\title{
Cardiac and Sympathetic Baroreflexes Remain Functional During Local Anesthesia With Vasoconstrictor-free Mepivacaine in Humans
}

\section{Chiaki Furutani}

Hiroshima University

Kazushige Isono

Matsumoto Dental University

Hironao Asahina

Matsumoto Dental University

Yudai Higuchi

Matsumoto Dental University

Kota Miyahara

Hiroshima University

Hironori Miyazaki

Hiroshima University

Yuki Oda

Hiroshima University

Masahiro Irifune

Hiroshima University

Sara Jarvis

Northern Arizona University

Tadashi Ogasawara

Matsumoto Dental University

Yoshiyuki Okada ( $\sim$ okay@hiroshima-u.ac.jp)

Department of Special Care Dentistry, Graduate School of Biomedical and Health Sciences, Hiroshima University, Hiroshima, Japan

\section{Research Article}

Keywords: baroreflex, sympathetic nerve activity, blood pressure, aging, local anesthesia

Posted Date: December 9th, 2020

DOI: https://doi.org/10.21203/rs.3.rs-118455/v1 
License: (c) (i) This work is licensed under a Creative Commons Attribution 4.0 International License. Read Full License 
Cardiac and sympathetic baroreflexes remain functional during local anesthesia with vasoconstrictor-free mepivacaine in humans

Chiaki Furutani $^{1}$, Kazushige Isono ${ }^{2}$, Hironao Asahina ${ }^{2}$, Yudai Higuchi ${ }^{2}$, Kota Miyahara ${ }^{1}$, Hironori Miyazaki ${ }^{1}$, Yuki Oda ${ }^{1}$, Masahiro Irifune ${ }^{3}$, Sara S. Jarvis ${ }^{4}$, Tadashi Ogasawara ${ }^{2}$, and Yoshiyuki Okada $^{1 *}$

${ }^{1}$ Department of Special Care Dentistry, Graduate School of Biomedical and Health Sciences, Hiroshima University, Hiroshima, Japan

${ }^{2}$ Department of Special Care Dentistry, Matsumoto Dental University, Shiojiri, Nagano, Japan ${ }^{3}$ Department of Dental Anesthesiology, Graduate School of Biomedical \& Health Sciences, Hiroshima University, Hiroshima, Japan

${ }^{4}$ Department of Biological Sciences, Northern Arizona University, Flagstaff, Arizona, USA

Running title: Mepivacaine and baroreflex sensitivity

Words: 1841, Tables: 1, Figures: 4

*Corresponding author: Yoshiyuki Okada, D.M.D., Ph.D

Department of Special Care Dentistry,

Graduate School of Biomedical and Health Sciences, Hiroshima University,

1-2-3 Kasumi, Minami-ku, Hiroshima, Japan 734-8553

Phone: +81-82-257-5788; Fax: +81-82-257-5789

Mail: okay@hiroshima-u.ac.jp 


\section{Abstract}

Local anesthesia with vasoconstrictor-free mepivacaine is known to not evoke pressor responses. However, it is unknown whether baroreflex function and blood pressure (BP) fluctuations are preserved by using mepivacaine. We tested the hypothesis that mepivacaine reduces baroreflex sensitivity (BRS) without changing its operating point. Beat-by-beat BP, heart rate (HR), and muscle sympathetic nerve activity (MSNA) were measured upon injection of either saline (CNT) or 3\% mepivacaine (MPV) in the apical regions of the premolars and around the mandibular foramen in 10 healthy young men [ $23 \pm 5$ (SD) years]. Cardiac and sympathetic BRSs were assessed by bolus injections of sodium nitroprusside followed by phenylephrine $\mathrm{HCl}$, and then determined from the slopes of regression lines between systolic BP and HR and between diastolic BP and MSNA, respectively. HR was significantly higher in MPV than CNT $(P<0.05)$, while there were no significant differences in MSNA, the operating points, or BP fluctuations between MPV and CNT (all P>0.05). Moreover, neither cardiac nor sympathetic BRS in CNT were altered by MPV $(-0.71 \pm 0.13$ vs. $-0.78 \pm 0.33$ beats $\cdot \mathrm{min}^{-1} \cdot \mathrm{mmHg}^{-1}, P=0.41 ;-0.98 \pm 0.35$ vs. $-0.92 \pm 0.16$ units $\cdot$ beat $^{-1}$. $\left.\mathrm{mmHg}^{-1}, P=0.73\right)$. Cardiac and sympathetic baroreflex functions were preserved and BP fluctuation may be well maintained under local anesthesia using vasoconstrictor-free $3 \%$ mepivacaine.

Keywords: baroreflex, sympathetic nerve activity, blood pressure, aging, local anesthesia 


\section{Introduction}

Due to the medical development and population aging, the prevalence of cardiovascular diseases has increased, and the possibility of using local anesthesia for surgical procedures in patients with decreased cardiovascular reserve has been increasing. This has raised the risk of fatal cardiovascular events associated with local anesthesia, especially during dental treatments $(1,2)$. Thus, it is important to evaluate the effects of local anesthetics on cardiovascular control systems for the safety of patients.

Most anesthetic solutions contain vasoconstrictors (e.g., adrenaline) to enhance the efficacy and prolong anesthesia duration. Despite their beneficial properties, it has been reported that administration of more than $40 \mu \mathrm{g}$ adrenaline affects cardiovascular parameters (3). For example, the plasma adrenaline concentration reached 2-fold greater than resting levels after administration of $1.8 \mathrm{ml}$ of lidocaine with 1:100,000 adrenaline (4), which was equivalent to that during moderate-to-heavy exercise (5). Therefore, its sympathoexcitatory effects may evoke cardiovascular mortality in patients at risk. The risks of using such vasoconstrictors should be weighed against the benefit, especially in cardiovascular patients.

Because mepivacaine, an amide-type local anesthetic, can sufficiently block somatosensory afferents and its half-life is long enough for almost all minor surgeries, such as dental procedures, even without vasoconstrictors, it is recommended for patients with cardiovascular diseases $(6,7)$. Indeed, intraoral administration of 3\% mepivacaine did not alter the average blood pressure (BP) per unit time in humans (8). However, the ability to maintain a steady BP should also be considered, because BP fluctuation beyond acceptable levels can cause cardiovascular events.

The baroreflex is a feedback system that maintains BP around its operating point (OP) by regulating cardiac output and total peripheral resistance through heart rate (HR) and muscle sympathetic nerve activity (MSNA) (9). The pressor responses to head-up tilt were 
greater in elderly blacks with lower baroreflex sensitivity (BRS) than in elderly whites (10), suggesting that blunted BRS contributes to greater BP responses during physiological stress. Higher plasma adrenaline concentration (11) and local anesthetics itself such as lidocaine (12) and bupivacaine (13) were reported to reduce BRS in animal studies. However, the effects of mepivacaine on baroreflex functions are unknown.

We hypothesized that using mepivacaine decreases BRS without changing the OP. Accordingly, we assessed the cardiac and sympathetic BRSs and the OPs by inducing BP alterations using intravenous injections of sodium nitroprusside (SNP) and phenylephrine $\mathrm{HCl}(\mathrm{PE})$ and compared responses to local anesthesia with 3\% mepivacaine versus saline of the same volume.

\section{Methods}

\section{Subjects}

Ten healthy young men who were non-smokers with no overt history of cardiovascular or any other chronic diseases participated in this study. None were currently taking any medications. All subjects provided written informed consent to a protocol performed according to the Declaration of Helsinki and approved by the Institutional Review Board of Matsumoto Dental University (0266). Their physical characteristics (means \pm SD) were $24 \pm 4$ years old, $176 \pm 6 \mathrm{~cm}$ tall, and $66.2 \pm 4.9 \mathrm{~kg}$ body weight.

\section{Measurements}

\section{Muscle sympathetic nerve activity:}

MSNA signals were obtained using microneurography $(14,15)$. In brief, a recording electrode (UNA35F4T; FHC, ME, USA) was placed in the peroneal nerve at the popliteal fossa, and a reference electrode was placed subcutaneously 2-3 cm apart from the recording 
electrode. The nerve signals were amplified (70,000 to 160,000-fold), band-pass filtered (700 to $2000 \mathrm{~Hz}$ ), full-wave rectified, and integrated with a resistance-capacitance circuit (time constant: $0.1 \mathrm{sec}$ ) to obtain a mean voltage neurogram. Criteria for adequate MSNA recording included pulse synchrony and facilitation during phase II of the Valsalva maneuver (15).

\section{Hemodynamics:}

HR was determined from the electrocardiogram (ECG) and arm cuff BP was measured by electronic sphygmomanometry with a microphone placed over the brachial artery on the left upper arm to detect Korotkoff sounds. Beat-by-beat BP was derived by finger photoplethysmography (Finometer; FMS, Amsterdam, The Netherlands), and the precision of beat-by-beat BP was verified by sphygmomanometry every minute.

\section{Protocol}

The experiment was performed in the morning $\geq 2 \mathrm{~h}$ after a light meal and $\geq 12 \mathrm{~h}$ after the last caffeinated or alcoholic beverage in a quiet, environmentally controlled chamber at an ambient temperature of $\sim 29^{\circ} \mathrm{C}$ with a relative humidity of $\sim 30 \%$. The subjects were placed in the supine position and an 18-gauge Teflon catheter (SR-FS1832; TERUMO, Tokyo) was inserted into the antecubital vein for bolus injections of vasoactive drugs. After a satisfactory nerve recording site was found, the subjects rested for more than 20 min before starting the measurements. They then blindly underwent two sequential trials, first under placebo injections with saline (CNT), then second under an alveolar nerve block with 3\% mepivacaine (MPV), which were separated by a 20-min rest. Each trial was consisted of a 5min baseline and 3-min measurements of HR and MSNA in response to vasoactive drugsinduced BP alterations. Throughout the entire procedure, respiratory rate was maintained at 
16 times/min and the waveforms were continuously monitored by a piezo respiratory belt transducer (MLT1132, AD Instruments, Dunedin, New Zealand).

\section{Local anesthesia with mepivacaine:}

In MPV, we infiltrated $1.8 \mathrm{ml}$ of $3 \%$ mepivacaine without vasoconstrictors into an area close to the mandibular foramen on the medial aspect of the mandibular ramus. We then injected another $1.8 \mathrm{ml}$ of the solution into the buccal sides of the root apexes of upper and lower premolars. In CNT, $1.8 \mathrm{ml}$ of saline solution was injected into each area in the same way as in MPV. The anesthetic effect was confirmed by stroking the gingival surface with a cotton ball and pricking it with a pin. If the depth of anesthesia was found to be insufficient, an additional amount of anesthetic solution was injected, but not beyond $300 \mathrm{mg}$ or $4.4 \mathrm{mg} / \mathrm{kg}$ mepivacaine in subjects weighing $<69 \mathrm{~kg}$ according to clinical practice guidelines (10).

\section{Baroreflex test with vasoactive drugs:}

The modified Oxford method was applied to determine cardiac and sympathetic BRSs, as reported previously (17). After baseline measurement, SNP $(100 \mu \mathrm{g})$ was injected through the intravenous catheter to decrease BP. HR and MSNA responses were measured for the following $1 \mathrm{~min}$. PE $(150 \mu \mathrm{g})$ was then injected to increase BP and the responses were measured for the following $2 \mathrm{~min}$.

\section{Data analysis}

Data were sampled at $625 \mathrm{~Hz}$ with a data acquisition system (PowerLab; ADInsturments, Dunedin, New Zealand). Offline data analyses were performed using signal-processing software (LabView; National Instruments, Austin, TX, USA). Beat-by-beat HR was calculated from the R-R interval. Beat-by-beat systolic BP (SBP) and diastolic BP (DBP) 
were obtained from the arterial pressure waveform. SBP and DBP fluctuations were expressed by variance and the coefficient of variance (CV). Sympathetic bursts were identified from the integrated neurogram by a computer program (18) and confirmed by an experienced microneurographer who was blinded to the interventions.

The integrated neurogram was normalized by assigning a value of 1000 to the largest amplitude of a sympathetic burst during the 5-min baseline (9). Burst area was measured as the area under the curve of each sympathetic burst of the normalized integrated neurogram. As quantitative indices, the number of bursts per minute (burst frequency), the number of bursts per 100 heartbeats (burst incidence), mean area per burst (mean burst area), and the area per beat (total MSNA) were calculated as follows:

- Burst incidence $=$ burst number $/ 100$ heartbeats [bursts $/ 100$ beats]

- Burst frequency $=$ burst number/min $[$ bursts $/ \mathrm{min}]$

- Mean burst area $=\sum_{k=1}^{\text {burstnumber }}$ area $(k) /$ burst number [units/burst]

- Total MSNA $=\sum_{k=1}^{\text {burstnumber }}$ area $(k) /$ heartbeats [units] $=$ mean burst area $\times$ burst incidence $/ 100$ [units/beat]

\section{Baroreflex sensitivity:}

Cardiac BRS was evaluated with HR response to SBP altered by bolus injections of SNP and $\mathrm{PE}$, as reported previously $(17,19)$. SBP and the corresponding HR were grouped into bins of 2-mmHg increments covering the lowest to highest SBP. To determine a pair of representative SBP and HR values for each bin, we adopted the average values of SBP and HR belonging to the bin. Cardiac BRS was assessed using the slope of the linear correlation between the representative HR and SBP after confirming that they were significantly correlated at R2>0.49 $(17,20)$. Sympathetic BRS was evaluated with MSNA response to 
DBP altered by SNP and PE injections $(9,14,17)$. DBP and the corresponding MSNA were grouped into bins of $3-\mathrm{mmHg}$ increments covering the lowest to highest DBP. To determine a pair of representative DBP and total MSNA values for each bin, we adopted the average values of DBP and total MSNA belonging to the bin. Sympathetic BRS was assessed using the slope of the linear correlation between the representative MSNA and DBP after confirming that $\mathrm{R} 2>0.25(14,21)$. The pooling procedure reduces the statistical impact of inherent beat-by-beat variability attributable to non-baroreflex influence (e.g., respiration) (17). Moreover, a statistical weighting was adopted to minimize the effect of minor variations in bin position on the slope relative to the number of cardiac cycles in the bins (9).

\section{Statistical analysis}

Data analysis was performed using SigmaPlot12 (Systat Software Inc, Chicago, IL). Values are expressed as means $\pm \mathrm{SD}$. The sample size was derived from power estimation in a previous paper studying the cardiovascular changes induced by local anesthesia with $2 \%$ lidocaine with adrenaline (8). MSNA indices, hemodynamics, and BRSs were compared between conditions [CNT vs. MPV] using paired t-tests. Correlation coefficients (r) between SBP and HR and between DBP and total MSNA were transformed by Z-score normalization before the comparison. The slopes of the regression lines between SBP and HR and between DBP and total MSNA were also compared intra-individually between CNT and MPV by using the permutation test. MSNA and hemodynamic responses to the modified Oxford method were analyzed using 2-way repeated measures ANOVA with factors for conditions, time, and the interaction [conditions $\times$ time]. The Holm-Sidak test was used as post-hoc for multiple comparisons. $P<0.05$ was considered statistically significant. 


\section{Results}

\section{Influences of MPV on cardiovascular variables}

Table 1 depicts cardiovascular and nervous variables, as well as their fluctuations, at the baseline in CNT and MPV. There were no significant differences in all variables $(P>0.05)$ between conditions, except for HR. HR was significantly higher during MPV than CNT $(P<0.001)$.

\section{Cardiovascular responses to vasoactive drugs}

Representative changes in HR and integrated MSNA in response to altered BP by bolus injections of SNP and PE for a subject were shown in Figure 1. HR and integrated MSNA increased as BP decreased, while both decreased as BP increased. The fall in SBP and DBP produced by SNP were similar during CNT and MPV $(P=0.833$ and 0.704$)$ and the rise in SBP and DBP produced by PE were also similar ( $P=0.404$ and 0.130$)$. The time dependent changes in these variables on average did not differ between conditions without any significant interactions between conditions $\times$ time (Figure 2).

\section{Cardiac baroreflex sensitivity}

Figure $\mathbf{3} \boldsymbol{A}$ demonstrates a typical example of the relationship between SBP and HR for a subject during CNT and MPV. As in the figure, the slope of HR/SBP, cardiac BRS, seemed to be similar between conditions. Cardiac BRS in 10 subjects are summarized on average in Figure $3 \boldsymbol{B}$. There was no significant difference between CNT and MPV ( $-0.71 \pm 0.13$ vs. $-0.78 \pm 0.33$ beats $\cdot \min ^{-1} \cdot \mathrm{mmHg}^{-1} ;$ mean difference $=0.07 ; 95 \%$ confidence interval $(\mathrm{CI})$ : -0.11 to $0.25 ; P=0.407)$. The slope of the regression line was intra-individually compared between CNT and MPV and it did not differ in 8 of 10 subjects. The linear regressions between HR and SBP had good correlation for all subjects in CNT [ $\mathrm{r}=0.82-0.98$ (range)] and 
MPV [r=0.83-0.98], and the values transferred by Z-normalization did not differ between the conditions $(P=0.740)$ (Figure 3C).

\section{Sympathetic baroreflex sensitivity}

Figure $4 \boldsymbol{A}$ demonstrates a typical example of the relationship between DBP and total MSNA for a subject during CNT and MPV and the slope of total MSNA/DBP, sympathetic BRS, seemed to be similar between conditions. Sympathetic BRS in 6 subjects whose MSNA detected appropriately are summarized on average in Figure $\mathbf{4 B}$. There was no significant difference between CNT and MPV $\left(-0.98 \pm 0.35\right.$ vs. $-0.92 \pm 0.16$ units $\cdot$ beat ${ }^{-1} \cdot \mathrm{mmHg}^{-1}$; mean difference $=0.06 ; 95 \% \mathrm{CI}:-0.45$ to $0.34 ; P=0.732$ ). The slope of the regression line was also intra-individually compared between CNT and MPV and it did not differ in any subjects (6/6). The linear regressions between total MSNA and DBP had good correlation for all subjects in CNT $[\mathrm{r}=-0.63$ to -0.88$]$ and MPV $[\mathrm{r}=-0.67$ to -0.95$]$, and the values transferred by Znormalization showed no difference between the conditions $(P=0.667)$ (Figure 4C).

\section{Discussion}

The major findings were: the OPs of the baroreflex remained unchanged in SBP and DBP during MPV; baroreflex control of MSNA at the OP was not affected, while HR was reset to a higher level; cardiac and sympathetic BRSs were maintained; and BP fluctuations did not differ from those during CNT. Thus, using vasoconstrictor-free mepivacaine has little effects on cardiovascular control.

The OP is determined by the cardiovascular center in the medulla oblongata based on information from central command $(22,23)$ and peripheral organs (e.g., chemoreceptor) (2227). In our study, as the same procedures were blindly applied to CNT and MPV, central command must be the same or affected equally between the conditions. Since the OP 
remained unchanged by MPV, MPV did not have any peripheral effects on the OP. This was consistent with the previous reports demonstrating that MPV without vasoconstrictors did not alter average cuff $\mathrm{BP}(7,8,28,29)$, which should converge to the OP. In contrast, MPV increased HR, due to BP reduction through vasodilation elicited by MPV (30), which may be immediately compensated by increased HR via the cardiac baroreflex with an unaltered OP. Higher HR increases oxygen demand of the heart, while vasodilation lowers afterload, resulting in little change in total cardiac workload. Thus, increased HR evoked by MPV with maintenance of the OP may not increase the cardiovascular risks, which was different from the simultaneous increases in HR and SBP developed with lidocaine with adrenaline (31) which is the most frequently used anesthetics for the minor surgeries.

Acute BP deviations from the safe range is one of the factors of cardiovascular events. A higher BP variability was related to increased mortality (127\%), and $\sim 40 \%$ were more likely to have a heart attack or a stroke (32). Therefore, the ability to maintain BP within the safe range is important, as well as the average BP. If BRS is low, BP fluctuations increase and BP can frequently surpass the upper limit of safe levels. The use of lidocaine caused the reduction of cardiac BRS (12). Bupivacaine also depressed cardiac BRS in the presence of atenolol, but not in the presence of methylatropine in rats (13), indicating that the inhibition of cardiac BRS by bupivacaine may involve the vagal pathway. Conversely, cardiac BRS did not differ between MPV and CNT in our study, suggesting that mucosal infiltration of MPV maintains vagal control of HR. Since baroreflex modulation of HR is known to be mainly associated with SBP (17), the unaltered cardiac BRS may maintain SBP control during MPV. Indeed, there were no differences in SBP fluctuation assessed with variance and CV between MPV and CNT.

The baroreflex has another important arch that controls BP through MSNA. BP is monitored by baroreceptors in the carotid sinus and the aortic arch via mechanical extension 
of the wall due to BP changes - the mechanical component of sympathetic baroreflex. When the baroreflex center detects the discrepancy between the BP in barosensory arteries and the OP, MSNA is regulated as an efferent signal to the peripheral vessels - the neural component. Changes in TPR via vasoconstriction based on MSNA will then correct $\mathrm{BP}$-peripheral transduction. Mepivacaine absorbed into blood stream may deploy inhibitory effects at the carotid sinus, nerve, and vascular muscle cells. Because it has been reported that the central arteries remained constricted, similar to basal tone levels, despite intraoral administration of MPV (33), MPV did not seem to affect vascular tone of the barosensory arteries. Because the magnitude of MSNA response to constant BP changes induced by vasoactive drugs was the same after oral injection of MPV and CNT, the neural component may not be affected by MPV. Collectively, sympathetic BRS assessed between DBP and MSNA functioned well during MPV. Conversely, the peripheral transduction may be inhibited because MVP induces vasodilation $(34,35)$. Hence, even if the preliminary changes in MSNA by baroreflex were the same as those with CNT, the magnitude of vasoconstriction may be attenuated in MPV. However, this inhibition appears to be compensated by an immediate increase in HR, which kept BP within the normal range. Because of the preserved functions of mechanical and neural components, combined with a compensatory increase in HR to overcome inhibited peripheral transduction, sympathetic BRS is deemed as being unchanged by MPV. Indeed, DBP fluctuation did not change with MPV when assessed with variance and CV.

High BP, large BP fluctuations, and sympathetic nerve activation are independent cardiovascular event risk factors (36). Even if BP was controlled with antihypertensives, sympathetic nerve activity was higher than the normal (37). Therefore, such cardiovascular changes should be avoided by optimizing local anesthesia especially in patients with small cardiovascular reserves. An unaltered BRS and a preserved OP without an upward shift of MSNA in MPV are very meaningful to maintain steady BP during surgery. These 
preliminary observations should to be confirmed in elderly patients and high-risk patients in future studies.

Limitations. First, it is possible that no difference in BRS between CNT and MPV may be a type II error due to a low statistical power. As no previous reports have investigated the effects of mepivacaine on cardiovascular variables, the minimum sample size was determined based on hemodynamic outcomes in a previous study evaluating the effects of lidocaine with adrenaline (8). Because the mean differences in BRSs were infinitesimal compared with the 95\% CI, we believe that BRSs remained unchanged (or negligible) in MPV. Second, all subjects were men. Because BRS was reported to change with the menstrual cycle in women (38), we only included men to avoid any effects of the menstrual cycle. Third, we did not randomize the order of CNT and MPV because of the long-lasting effects of anesthesia. Since, we did not find any differences in hemodynamics, including BRS, the adaptation, fatigue, or central command in the second bout of baroreflex assessment was unlikely to affect BRS.

In summary, vasoconstrictor-free 3\% mepivacaine has little or no impact on the cardiac and sympathetic BRSs or the OPs. The intact neurogenic BP regulation during local anesthesia with mepivacaine may be preferred in high-cardiovascular risk patients.

\section{Data Availability}

The original recorded waveforms and datasets generated during the current study are available from the corresponding author on reasonable request. 


\section{References}

1. Y. Okada, H. Suzuki, I. Ishiyama, Fatal subarachnoid haemorrhage associated with dental local anaesthesia. Aust Dent J 34, 323-325 (1989).

2. J. Jage, Circulatory effects of vasoconstrictors combined with local anesthetics. Anesth Pain Control Dent 2, 81-86 (1993).

3. F. Morady et al., Electrophysiologic effects of epinephrine in humans. J Am Coll Cardiol 11, 1235-1244 (1988).

4. A. G. Tolas, A. E. Pflug, J. B. Halter, Arterial plasma epinephrine concentrations and hemodynamic responses after dental injection of local anesthetic with epinephrine. $J \mathrm{Am}$ Dent Assoc 104, 41-43 (1982).

5. P. E. Cryer, Physiology and pathophysiology of the human sympathoadrenal neuroendocrine system. $N$ Engl J Med 303, 436-444 (1980).

6. M. Daublander, R. Muller, M. D. Lipp, The incidence of complications associated with local anesthesia in dentistry. Anesth Prog 44, 132-141 (1997).

7. K. Replogle et al., Cardiovascular effects of intraosseous injections of 2 percent lidocaine with 1:100,000 epinephrine and 3 percent mepivacaine. $J$ Am Dent Assoc 130, 649-657 (1999).

8. A. G. Mohammad, Y. Javad, G. Z. Arezo, A. A, Comparison of Heart Rate and Blood Pressure administration of anesthesia agent with and without. Int J Curr Res Aca. 2, 153$158(2014)$.

9. J. R. Halliwill, Segregated signal averaging of sympathetic baroreflex responses in humans. J Appl Physiol (1985) 88, 767-773 (2000).

10. Y. Okada et al., Elderly blacks have a blunted sympathetic neural responsiveness but greater pressor response to orthostasis than elderly whites. Hypertension 60, 842-848 (2012). 
11. T. Kawada et al., Acute arterial baroreflex-mediated changes in plasma catecholamine concentrations in a chronic rat model of myocardial infarction. Physiol Rep 4, (2016).

12. I. Yoneda, M. Nishizawa, K. T. Benson, T. L. Chaffee, H. Goto, Attenuation of arterial baroreflex control of renal sympathetic nerve activity during lidocaine infusion in alphachloralose-anesthetized dogs. Acta Anaesthesiol Scand 38, 70-74 (1994).

13. K. S. Chang, D. R. Morrow, K. Kuzume, M. C. Andresen, Bupivacaine inhibits baroreflex control of heart rate in conscious rats. Anesthesiology 92, 197-207 (2000).

14. G. Sundlof, B. G. Wallin, Human muscle nerve sympathetic activity at rest. Relationship to blood pressure and age. J Physiol 274, 621-637 (1978).

15. A. B. Vallbo, K. E. Hagbarth, H. E. Torebjork, B. G. Wallin, Somatosensory, proprioceptive, and sympathetic activity in human peripheral nerves. Physiol Rev 59, 919-957 (1979).

16. S. F. Malamed, Handbook of local anesthesia. (Elsevier/Mosby, St. Louis, Mo., ed. 5th, 2004), pp. xiii, 399 p.

17. L. Rudas et al., Human sympathetic and vagal baroreflex responses to sequential nitroprusside and phenylephrine. Am J Physiol 276, H1691-1698 (1999).

18. J. Cui, T. E. Wilson, C. G. Crandall, Baroreflex modulation of muscle sympathetic nerve activity during cold pressor test in humans. Am J Physiol Heart Circ Physiol 282, H17171723 (2002).

19. T. J. Ebert, A. W. Cowley, Jr., Baroreflex modulation of sympathetic outflow during physiological increases of vasopressin in humans. Am J Physiol 262, H1372-1378 (1992).

20. H. S. Smyth, P. Sleight, G. W. Pickering, Reflex regulation of arterial pressure during sleep in man. A quantitative method of assessing baroreflex sensitivity. Circ Res 24, 109$121(1969)$. 
21. B. M. Eicke, K. Milke, T. Schlereth, F. Birklein, Comparison of continuous wave Doppler ultrasound of the radial artery and laser Doppler flowmetry of the fingertips with sympathetic stimulation. J Neurol 251, 958-962 (2004).

22. L. B. Rowell, Human cardiovascular control. (Oxford University Press, New York, 1993), pp. xv, 500 p.

23. L. B. Rowell, J. T. Shepherd, American Physiological Society (1887- ), Exercise: regulation and integration of multiple systems. Handbook of physiology (Published for the American Physiological Society by Oxford University Press, New York, 1996), pp. xiii, $1210 \mathrm{p}$.

24. G. M. Goodwin, D. I. McCloskey, J. H. Mitchell, Cardiovascular and respiratory responses to changes in central command during isometric exercise at constant muscle tension. J Physiol 226, 173-190 (1972).

25. S. Ogoh et al., Role of central command in carotid baroreflex resetting in humans during static exercise. J Physiol 543, 349-364 (2002).

26. J. H. Mitchell, M. P. Kaufman, G. A. Iwamoto, The exercise pressor reflex: its cardiovascular effects, afferent mechanisms, and central pathways. Annu Rev Physiol 45, 229-242 (1983).

27. L. B. Rowell, D. S. O'Leary, Reflex control of the circulation during exercise: chemoreflexes and mechanoreflexes. J Appl Physiol (1985) 69, 407-418 (1990).

28. B. Ezmek, A. Arslan, C. Delilbasi, K. Sencift, Comparison of hemodynamic effects of lidocaine, prilocaine and mepivacaine solutions without vasoconstrictor in hypertensive patients. J Appl Oral Sci 18, 354-359 (2010).

29.G. N. Smith, D. H. Pashley, Periodontal ligament injection: evaluation of systemic effects. Oral Surg Oral Med Oral Pathol 56, 571-574 (1983). 
30. H. J. Sung et al., Mepivacaine-induced contraction is attenuated by endothelial nitric oxide release in isolated rat aorta. Can J Physiol Pharmacol 90, 863-872 (2012).

31. W. E. Clutter, D. M. Bier, S. D. Shah, P. E. Cryer, Epinephrine plasma metabolic clearance rates and physiologic thresholds for metabolic and hemodynamic actions in man. J Clin Invest 66, 94-101 (1980).

32. M. K. Kim et al., Associations of Variability in Blood Pressure, Glucose and Cholesterol Concentrations, and Body Mass Index With Mortality and Cardiovascular Outcomes in the General Population. Circulation 138, 2627-2637 (2018).

33. K. Satoh et al., The effect of mepivacaine on swine lingual, pulmonary and coronary arteries. BMC Anesthesiol 15, 101 (2015).

34. M. R. Blair, Cardiovascular pharmacology of local anaesthetics. Br J Anaesth $\mathbf{4 7}$ suppl, 247-252 (1975).

35. H. H. Lindorf, Investigation of the vascular effect of newer local anesthetics and vasoconstrictors. Oral Surg Oral Med Oral Pathol 48, 292-297 (1979).

36. N. Charkoudian, J. A. Rabbitts, Sympathetic neural mechanisms in human cardiovascular health and disease. Mayo Clin Proc 84, 822-830 (2009).

37. Y. Okada et al., Chronic renin inhibition lowers blood pressure and reduces upright muscle sympathetic nerve activity in hypertensive seniors. $J$ Physiol 591, 5913-5922 (2013).

38. R. Koifman, L. Dayan, J. N. Ablin, G. Jacob, Cardiovascular Autonomic Profile in Women With Premenstrual Syndrome. Front Physiol 9, 1384 (2018). 


\section{Acknowledgments}

We are grateful to the study volunteers for their participation. This work was supported by Ryokufu-kai encouragement award of educational research and the fund of Nagano Prefecture to promote scientific activity.

\section{Author contributions}

C. F contributed to conception and design of the experiments; acquisition, analysis and interpretation of data; and drafting the article and revising it. K. I, H. A, Y. H and K. M contributed to acquisition and analysis of data and revising the article. H. M, Y. O, M. I, and

T. O contributed to interpretation of data and revising the article. Y. O contributed to conception and design of the experiments; acquisition, analysis and interpretation of data; and revising the article critically for important intellectual content. All authors gave their final approval and agree to be accountable for all aspects of the work.

\section{Conflicts of interest}

The authors declare no competing interests. 


\section{Figure Legends}

Figure 1. Representative data of heart rate (HR), blood pressure (BP), and muscle sympathetic nerve activity (MSNA) in response to vasoactive drug infusions, SNP and PE, for a subject.

Figure 2. Hemodynamic responses in HR, systolic BP (SBP), diastolic BP (DBP), and MSNA to infusions of SNP and PE during control (CNT) and mepivacaine (MVP) conditions. Values are mean \pm SD.

Figure 3: Typical examples of the relationships between and between SBP and HR for a subject $(\boldsymbol{A})$, cardiac BRS $(\boldsymbol{B})$ and correlation coefficient (r) of the relationship between SBP and HR, which was transferred by Z-normalization $(\boldsymbol{C})$. Values are mean $\pm \mathrm{SD}$.

Figure 4. Typical examples of the relationships between and between DBP and MSNA for a subject $(\boldsymbol{A})$, sympathetic baroreflex sensitivity (BRS) $(\boldsymbol{B})$ and correlation coefficient $(\mathrm{r})$ of the relationship between DBP and MSNA, which was transferred by Z-normalization $(\boldsymbol{C})$. Values are mean \pm SD 
Table 1. Cardiovascular variables and their fluctuations during baseline

\begin{tabular}{lcc}
\hline & CNT & MPV \\
\hline SBP, mmHg & $125 \pm 8$ & $125 \pm 5$ \\
DBP, mmHg & $59 \pm 13$ & $60 \pm 14$ \\
HR, beats $\cdot$ min $^{-1}$ & $56 \pm 8$ & $61 \pm 12 *$ \\
Total MSNA, units $\cdot$ beat $^{-1}$ & $19 \pm 2$ & $21 \pm 3$ \\
\hline Variance (SBP), mmHg & $35 \pm 22$ & $23 \pm 11$ \\
Variance (DBP), mmHg & $9 \pm 4$ & $11 \pm 11$ \\
CV (SBP), \% & $4 \pm 1$ & $5 \pm 1$ \\
CV (DBP), \% & $4 \pm 1$ & $5 \pm 1$ \\
\hline
\end{tabular}

Values are means \pm S.D. CNT, saline; MPV, 3\%mepivacaine; SBP, systolic blood pressure; DBP, diastolic blood pressure; HR, heart rate; MSNA, muscle sympathetic nerve activity; ${ }^{*}, \mathrm{P}<0.05$. 


\section{Figures}

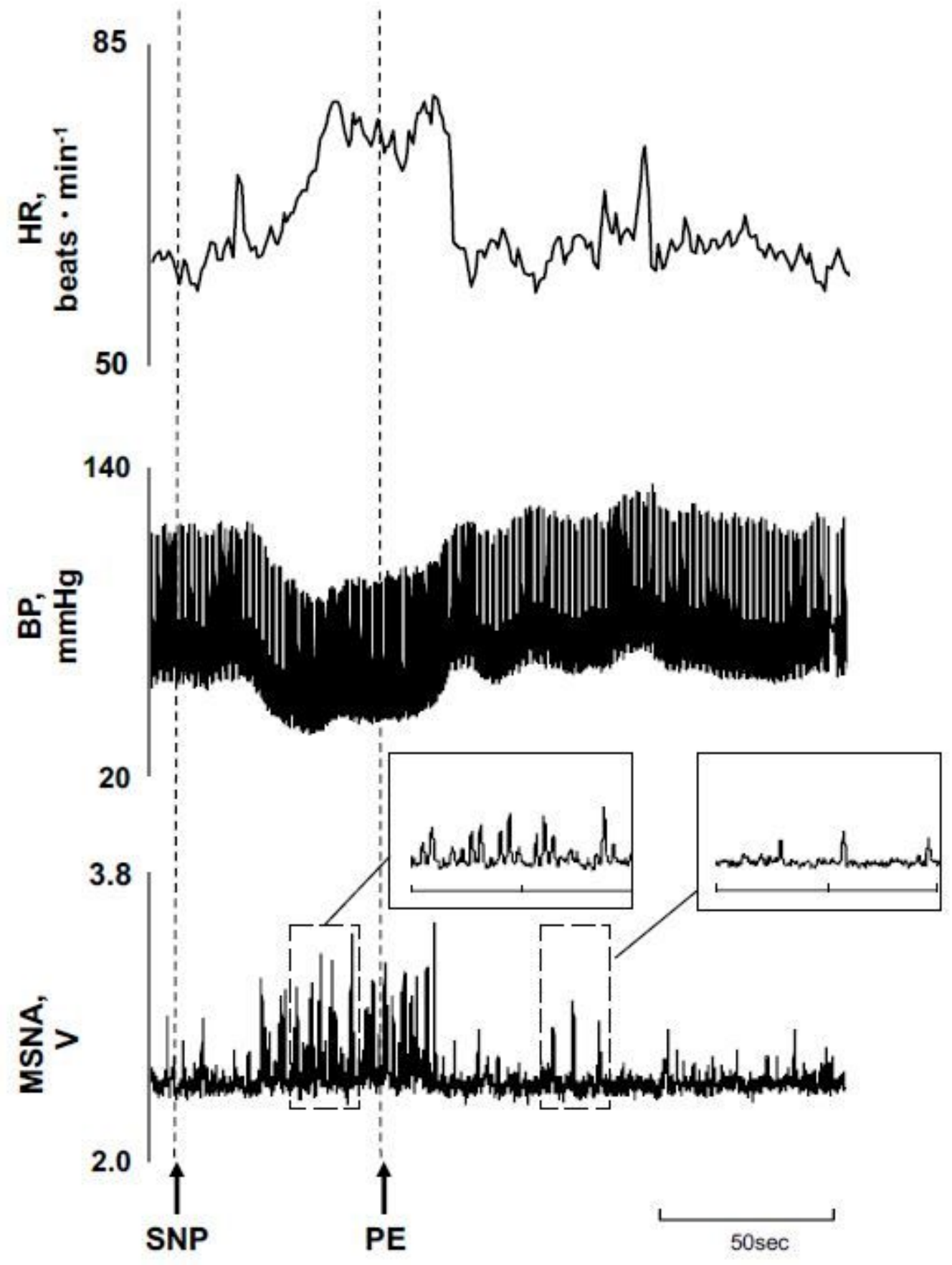

Figure 1

Representative data of heart rate (HR), blood pressure (BP), and muscle sympathetic nerve activity (MSNA) in response to vasoactive drug infusions, SNP and PE, for a subject. 


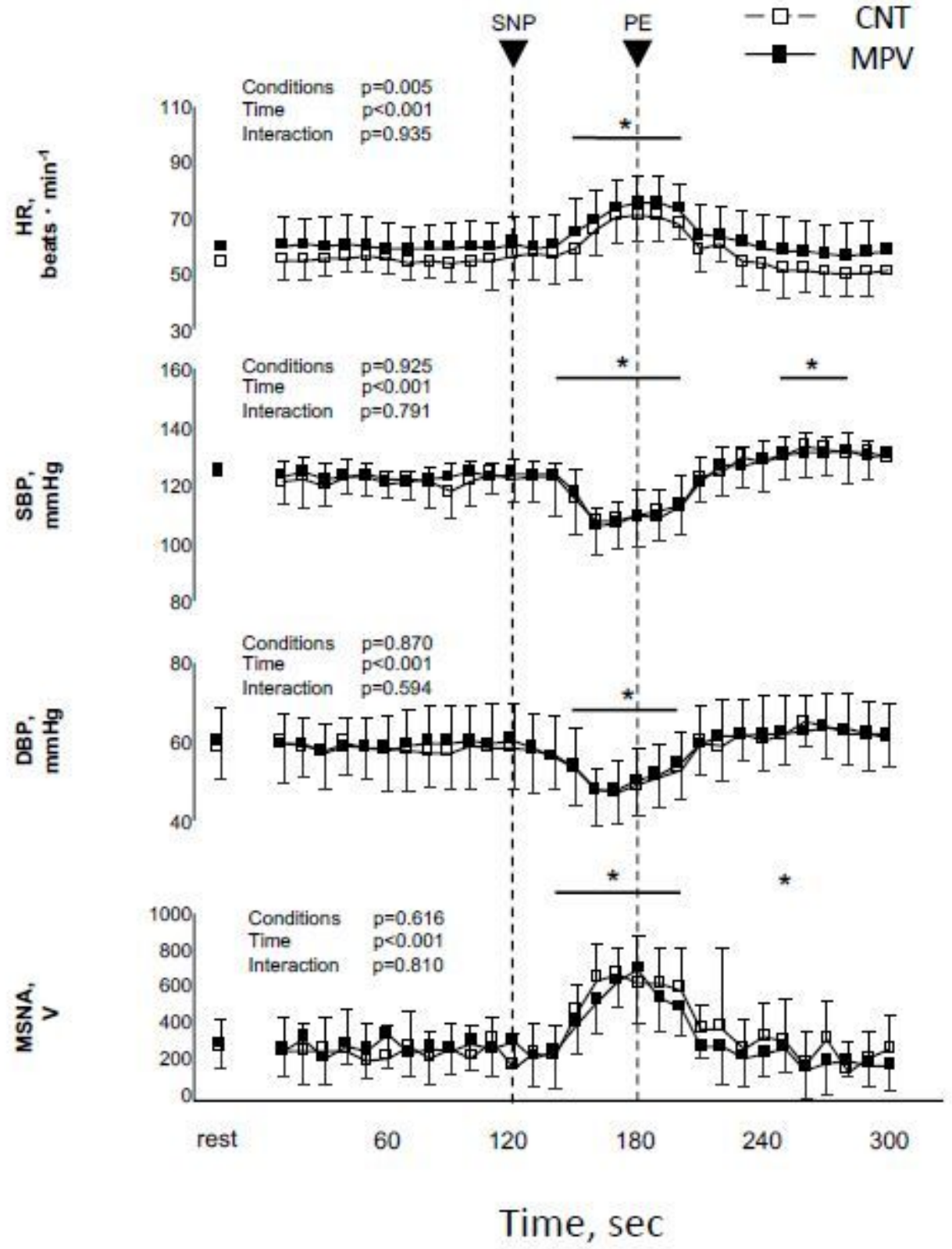

Figure 2

Hemodynamic responses in HR, systolic BP (SBP), diastolic BP (DBP), and MSNA to infusions of SNP and PE during control (CNT) and mepivacaine (MVP) conditions. Values are mean $\pm S D$. 


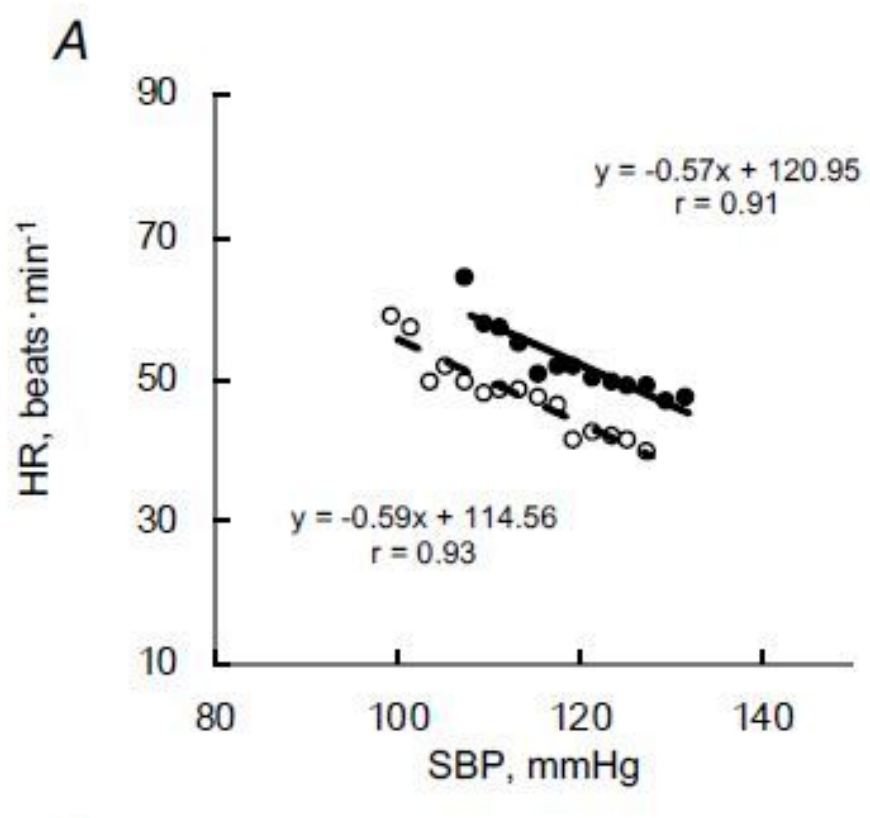

$B$
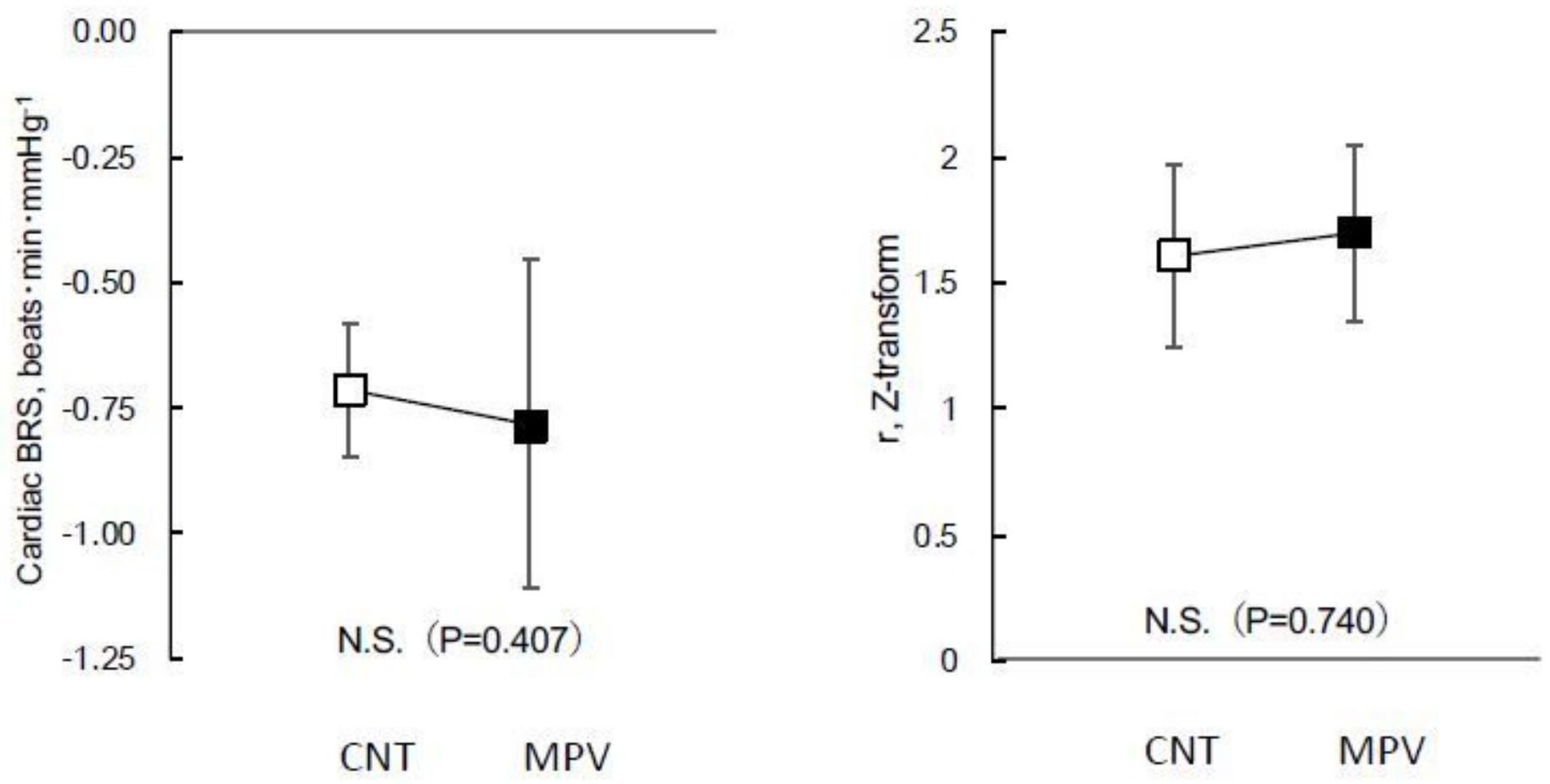

Figure 3

Typical examples of the relationships between and between SBP and HR for a subject (A), cardiac BRS (B) and correlation coefficient ( $r$ ) of the relationship between SBP and HR, which was transferred by Znormalization (C). Values are mean \pm SD. 


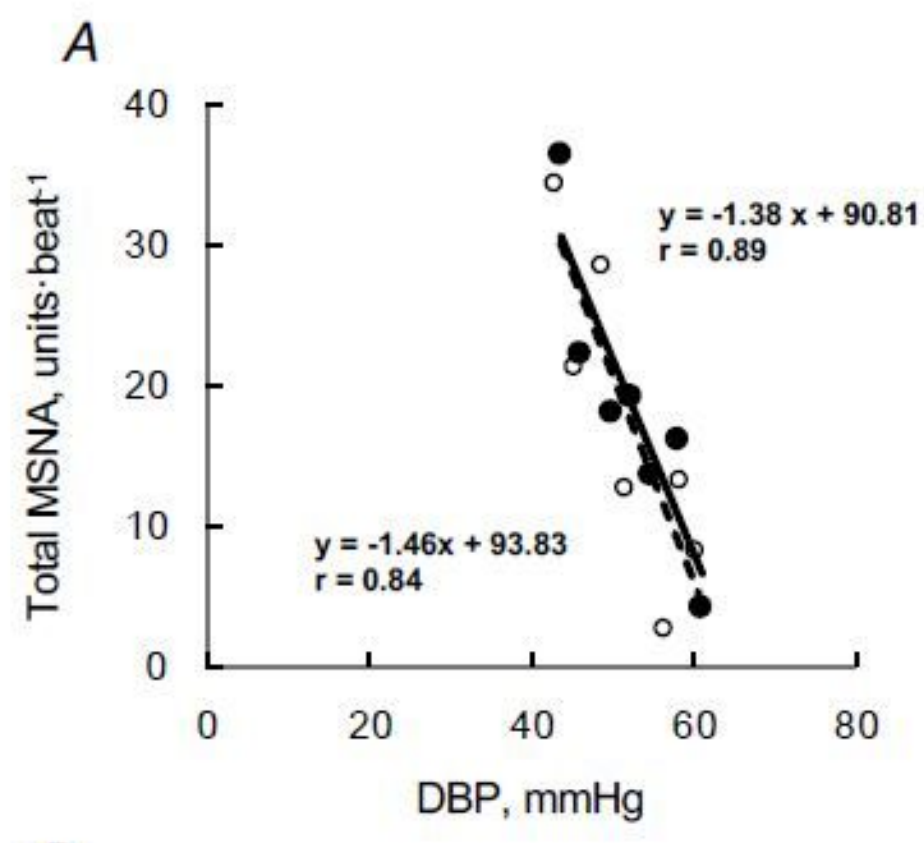

$B$

C
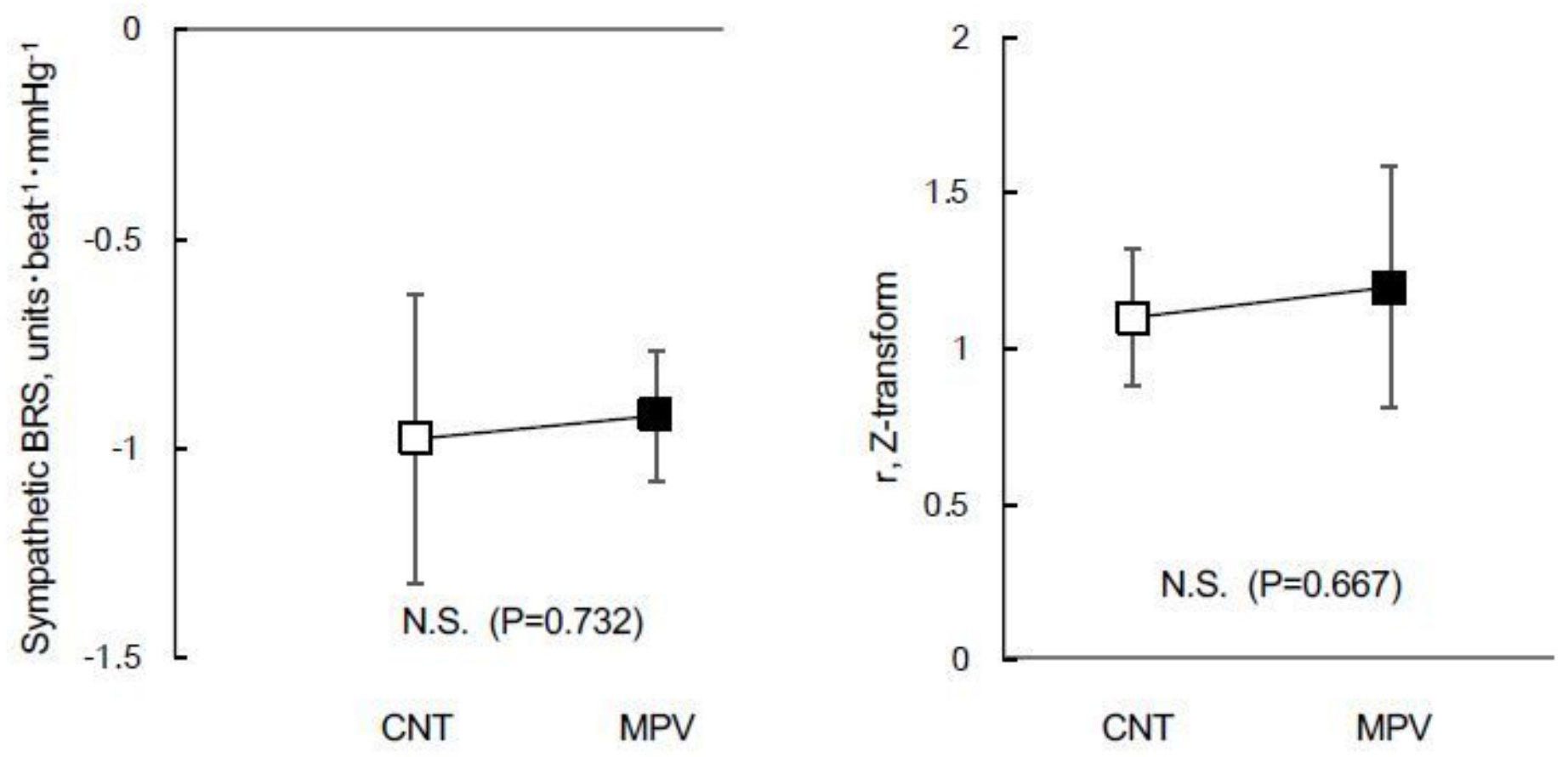

Figure 4

Typical examples of the relationships between and between DBP and MSNA for a subject (A), sympathetic baroreflex sensitivity (BRS) (B) and correlation coefficient ( $r$ ) of the relationship between DBP and MSNA, which was transferred by Z-normalization (C). Values are mean \pm SD 